\title{
Rites of passage: Identity and the training of clinical psychologists in the current South African context
}

\author{
Amanda Kottler* \\ 1 Mobema Court, Derry Street, Vredehoek, 8001, South Africa \\ (Formerly at the Child Guidance Clinic, University of Cape Town)
}

\section{Sally Swartz}

Child Guidance Clinic, University of Cape Town, South Africa

This article attempts to make sense of the clinical psychology training experience, as a negotiation of personal and professional identity. It suggests that the training, particularly in the first year, is a rite of passage, in many respects similar to an initiation process. Rites of passage can be seen as processes marking shifts in status and social identity. In the case of training to be a psychologist this change involves the movement from lay to professional status. There are three phases involved in the transitional process. The first involves separation from other groups of postgraduate students, and also in different ways from friends and family through involvement in course material that is often both esoteric and confidential. The second phase is marked by a fluid and often confusing marginal state between student and professional identity. The third phase involves a reintegration back into society as a fully-fledged professional, once the training is over. This article focuses on the ambiguities of the marginal state as the primary identity position during training, and explores the implications of this, both for those being trained and for trainers.

* To whom correspondence should be addressed

Training to become a clinical psychologist is widely known to be an arduous process. It demands that trainees become intimately involved in the pain, conflicts, disappointments and hardships of the lives of people whose mental health in some way is in jeopardy. It also demands that trainees examine their own lives, and negotiate their way towards a professional identity that will allow them simultaneously to grapple 
with the pain of others in a mindful way and to protect themselves from this activity's potentially debilitating effects. Many students have held the training as a somewhat idealised life ambition. Once involved in the academic, clinical and personal pressures of the training, however, many begin to question themselves and their work, and to wonder whether they have made a wise choice of career. Some contemplate withdrawing from the training and a few do. Sometimes long-standing relationships flounder or end and it is common for trainees to experience periods of intense anxiety or depression.

This article attempts to make sense of the training experience as a complex negotiation between personal identity and social or professional identity. Group membership provides individuals with a definition of themselves as actors in the social world (Tajfel, 1979). This article will illustrate ways in which becoming a member of the professional psychology community offers trainees a variety of definitions of their emerging roles, many of which will require them to interrogate the roles they have played in their families, with their friends, and in their life as students. Paradoxically, the sense of who they are, as unique individuals, and their willingness to be open to exploration of often private feelings, are a part of negotiating membership to the professional group. Intimacy and readiness to engage in personal disclosure without defensiveness is highly salient to the training. This is in contrast to many other forms of professional training, in which technical, academic or even artistic skills are more salient than personal characteristics. The training itself, quite apart from family and friendship commitments, demands that trainees occupy multiple roles, and this, too, is often stressful (Barnett \& Hyde, 2001; Stryker \& Burke, 2000).

Thus, entry into professional psychology training impacts upon definitions of self, causing emotional and cognitive turbulence, at least for the period of the training, and sometimes longer. Tensions arise from conflicts between a continuous sense of selfhood, a position in a social order in terms of class, gender, sexuality, race and culture, and the demands and prohibitions of a profession that has rigorous rules about how relationships should be conducted. This article suggests that the training, particularly in the first year, is a rite of passage of the kind that 'accompanies every change of place, state, social position and age' of an individual (Van Gennep, in Wengle, 1987, p. 357) and which are 'most conspicuous at the times of critical transitions in life, that is birth, puberty, marriage, and death' (Wengle, 1987, p. 357).

\section{DATA AND ANALYSIS}

The data for this article derives from 20 years of experience of the University of Cape Town's (UCT) clinical psychology training, first as trainees and later as trainers. It is a case study drawing on direct experience, and also students' accounts of their daily lives in the training, written in formal and informal feedback, throughout the year. Documentation from quarterly feedback sessions and from students' feedback to staff at the end of each year is one source of data; three years of weekly notes from 
students on their experience in the form of an ongoing logbook is another. The case study attempts to generalise across diversity to describe repeated patterns. The description that follows has therefore used the data to isolate broad thematic structures. Unique individual experience is not discussed.

Case studies may focus on a single individual, or a community, an institution, or a number of individuals engaged in a 'sequence of activities' (Clyde Mitchell 2000, p. 169). Clyde Mitchell (2000, p. 169) argues that their purpose is to organise accumulated observations 'to support theoretical conclusions'. This case study is a structuralist analysis, in the sense that it uses the stages described as belonging to rites of passage, and applies these to a community of psychology trainees. It is largely an examination of the UCT clinical psychology course. Where appropriate, it draws on the experience of a wider community of South African psychology training courses. These experiences have been garnered from joint teaching, external examination and many collegial discussions.

\section{THE TRAINING PROGRAMME}

Master's-level clinical psychology training in South Africa aims to produce clinical psychologists with a basic knowledge of diagnostic and assessment skills, together with a range of possible psychological interventions informed by a variety of theoretical frameworks. It increasingly demands a thorough appreciation of the particular challenges facing South African practitioners, who often find themselves working in multilingual and drastically under-resourced communities, in which even the most basic services (e.g. access to electricity and running water, availability of primary health care and adequate schooling) are threadbare. It has been widely acknowledged that British and American models of clinical psychology training, while useful in many respects, do not hold all the answers for psychologists working in Africa. Much progress has been made in constructing models of training appropriate to South Africa's needs (e.g. Duncan, van Niekerk, de la Rey \& Seedat, 2001; Seedat, Duncan \& Lazarus, 2001; Suffla, Stevens \& Seedat, 2001; Swartz, 1998; Swartz, Gibson \& Gelman, 2002).

Clinical training at UCT has three major components. The first component, which is introduced in the first year, is made up of a programme of seminars, constituting an intensive preparation for clinical work, and these are complemented by closely supervised experience with a limited number of clients. The second component is introduced in the second year and entails an internship in a mental hospital setting. Trainees carry a larger caseload and are exposed to a range of mental health settings, including acute admission wards, neurology and forensic wards, and therapeutic inpatient programmes. In addition to this, they are expected to have some experience in primary health care clinics or in non-governmental organisations responding to situations of ongoing trauma. The third component of the course, generally completed after the internship year, is a research dissertation.

Clinical training is associated with a number of marked transitions: from student to trainee; from trainee with no case load to trainee working clinically with clients; 
from university-based trainee to intern; from intern to dissertation writer; and finally, from trainee to registered psychologist qualified to practice. Each transition requires practical and emotional adjustments to new demands. It is with the transitions of the first year, and their similarity to a rite of passage that this article is concerned.

\section{INITIATION RITES AND THE TRANSFORMATIONAL PASSAGE}

In considering initiation rites as the main feature of instruction within particular smallscale societies, Turner $(1967$, p. 93) introduces the idea of 'interstructural' human beings embarking upon what Van Gennep (in Turner, 1967, p. 94) has described as 'rites of passage', which 'accompany every change of place, state, social position and age'. The model proposes that there are three phases involved in the transitional process: separation, marginality and reintegration.

The separation phase is filled with symbolic behaviour signifying to the wider society that the individual or group has become detached 'from an earlier fixed point in the social structure, from a set of cultural conditions (a "state") or from both' (Turner, 1967, p. 94). Thus, initiates are removed from society, as it is known to them. They are taken to a place outside their community where they live with their instructors for a prescribed period. In the wilderness, they are unconstrained by the norms and conventions of society. In direct contrast to the predictability of the social life initiates have known thus far, it is a world characterised by feelings of flux and instability.

In the marginal phase Turner suggests that 'the characteristics of the ritual subject (the "passenger") are ambiguous; he passes through a cultural realm that has few or none of the attributes of the past or coming state' (Turner, 1967, p. 94). Turner suggests that it is a nebulous period, characterised by an absence of not only everything that has been familiar in the past, but also of all that is to come in the future (Wengle, 1987). Because it is a timeless, invisible, secret and hidden phase, it is difficult to describe. Much of what happens to initiates, after the early rituals around separation have occurred, is experiential, considered secret and is not therefore documented. Similarly, much of the experience of UCT students (which feels extremely private to each of them) has remained undocumented.

In an initiation process, initiates are reintegrated into society as adults with a new role and social identity. However, and this is significant for the purposes of our article, this happens only after a well-marked and protracted phase and only once they are in a 'relatively stable state once more' (Turner, 1969, p. 95, our italics).

The literature describes the marginal phase as one which entails a symbolic death - each initiate experiences the annihilation of his or her own sense of identity and all that he or she has come to know about himself or herself and the way he or she operates in the world. With this lost, initiates have to find a way of constructing a new identity - a painful process of re-birth, during which time they are introduced to some of the basic assumptions of the culture into which they will pass (Wengle, 
1987). Perhaps the most difficult aspect of this conflict-filled demand stems from the idea that in the interstructural, marginal phase, initiates become structurally invisible and no longer classifiable. Subjectively speaking, they become 'corpses' in relation to their past status and 'infants' in relation to their future status (Wengle, 1987, p. 157). It is with the comparable tension between the marginal state and progression towards reintegration, between not knowing and also being expected to know that this article is concerned.

\section{Clinical training as a rite of passage}

Although clinical trainees are not actually taken to a wilderness outside of their community, there are other ways in which they are detached from a larger body of postgraduate students. Sometimes they are housed geographically in clinics or university offices that separate them from undergraduates and other postgraduate students. Further, unlike previous periods of study, they are often expected to be in attendance at seminars, supervision or clinical appointments from 8:30 to 17:00 or 18:00. This enforces a temporal separation from family and friends, and is something trainees often remark on. They comment wryly that they feel as if they see more of their fellow trainees and lecturers than their partners. This is also the time during which family members and friends begin to voice complaints, or express anxiety about the trainee's relative absence in their lives - an absence not only in terms of time, but also of a new and more hidden preoccupation.

Perhaps the most important means of transporting clinical trainees into a wilderness is simply through secrecy. Many of the experiences into which trainees are thrown cannot be discussed outside of the training for reasons of confidentiality. Beyond that, the nature of clinical work, often difficult for the outsider to understand without some firsthand experience, becomes shrouded in strange language and strong feeling, and this enforces a mental separation from others in a wider community of students. The split dividing intimate experience at work from the ordinary intimacies of friendship and family interrogates and rearranges social relationships; friendships in which trainees were cast in the role of offering support and advice tend to dwindle.

During the early phases of training, students are introduced to a different world to the one (or ones) in which they have grown up. Ordinary, everyday social encounters and ways of politely relating, according to well-accepted but unspoken conversational rules are challenged. In what feels to some like a nurturing, contained and almost therapeutic space, trainees and staff begin to explore a range of very personal issues. These include the reasons for their choice of clinical psychology as a career, and aspects of their family background and relationships. This challenges aspects of personal identity, and once-stable assumptions about self and the world may begin to be questioned. In preparation for their initial meeting with their first clients, students are introduced to a number of widely different, and sometimes conflicting and contradictory, models of clinical work. 
Despite the bridges that staff attempt to build between family and individual interventions, group work and community projects, the ideological schisms between different branches of the profession are profoundly unsettling for beginner therapists in search of solid ground. Disorientating too are the leaps between purely academic exercises and intimate personal discussion, between theoretical debate and exposure to examples of clinical work, observed from behind a one-way mirror. This phase could be seen as the start of an 'interstructural situation' (Turner, 1969, p. 93), which continues into, and throughout, the second 'marginal' phase. Models of clinical work become the 'sacra', the symbolic template of the whole system of beliefs and values in a given culture (Turner, 1967, p. 108). This is the knowledge that will ultimately make initiates true members of their society.

The second phase of the rite of passage, that of living a destabilised existence in the margins between two identities, could be argued to continue for at least the first year of training, during which time trainees are students (but unlike other students) and also clinicians (albeit under supervision) working directly with clients, but with none of the 'adult' status of qualified and registered clinicians.

The marginal state of the trainees appears to make a substantial shift during the course of the internship year. The anxieties and sense of fragmentation that accompany the first year of training give way to an increasing experience of competence, of having achieved membership of an established social group and, most importantly, of beginning to achieve adult status. There are a number of reasons for this. Firstly, the interns receive a salary, and this marker of the shift from student to paid employee contributes substantially to their sense of membership of the profession. Secondly, in a variety of busy placements, they take their place as members of a team and the focus of the work is often far more on service than on training. Moreover, other members of the psychiatric team look to intern psychologists to provide specialised skills and knowledge, particularly in the areas of psychometric assessment, and a wide range of psychotherapeutic interventions. A growing proportion of routine work is simply checked rather than discussed at any length. The severity of many cases seen in the internship year adds to the burden of interns' responsibility, but also is helpful in contributing to a sense of achieving a professional identity. The accumulation of many hours of clinical work, of making informed decisions about often very difficult cases, and of being treated as a full member of a professional team takes interns to an emotional position far removed from the turmoil and uncertainty of the first year of training. The gain in professional identity also marks a shift in social status.

\section{The death, the dying and the struggle for identity}

The extent to which clinical trainees feel helpless, dependent and estranged from past competencies during what we have termed the marginal phase of the ritual journey to professional status, is a recurring theme in personal accounts of the process. 
Trainees, even those with years of counselling experience in other settings, find themselves in search of what they consider to be 'real' counselling or therapeutic skills, so thoroughly have they assumed that what they were doing in the past was no more than a makeshift approximation. Trainees often feel suddenly cast adrift, expected not only to behave with clients as if they are confident professionals, but also to confront and deal with personal change and growth, which are likely to be overwhelming for even an experienced clinician. This is a tall order, given that studies have suggested that there is a 'breakdown of cognitive schematic frameworks [in the Piagetian sense] during this [marginal] stage' (McManus, in Wengle, 1987, p. 358). This is possible because of their isolation from their normal surroundings, as well as the extreme physical and mental pressure placed on them. These pressures eventually 'destroy the individual's sense of personal, inner continuity and plunge him into emotional collapse' (Wengle, 1987, p. 359).

Thus, at this stage, initiates (and clearly, clinical trainees will identify with this) are in a state of profound ambiguity, paradox and contradiction. Many trainees have been high achievers with a need for order, and work habits that suggest their capacity to cope with the 'essentially self-teaching aspect of training' (Quinodoz, 1996, p. 494). Then they are confronted in the training with an inner journey, with clients and with therapeutic challenges (both personal and professional) not entirely under their control. The phase feels psychologically messy and unstructured. As one trainee put it: 'I have a feeling right at the moment of exploding. Going right into pieces.'

Not every trainee will experience the course as traumatic. However, all are likely to experience some turbulence in their sense of social identity and each will engage in his or her own idiosyncratic protective behaviours during training. The extent of the struggle involved for each will depend largely on what past experience each brings into the course and how his or her particular dynamics manifest themselves at this point in the training process. How each trainee psychologist is affected will depend on the state of his or her self as he or she enters the course and the particular relationships that constellate his or her social and personal identity.

Some clinical trainees have reported feelings of profound alienation as a result of 'difference' (dynamically influenced, but also contextually valid). We are referring, for example, to feeling like a 'token black' in a predominantly white class, with an all-white staff complement, at a historically white university or, to feeling like the 'token lesbian' in a predominantly 'straight' class, with a predominantly 'straight' staff complement. The use of theories founded in a body of literature originating from within predominantly white, middle-class, heterosexual-as-norm discourses compounds the feelings of alienation. In these situations, it is social identity that becomes the focus of painful experiences of exclusion and voicelessness. These experiences reiterate South Africa's history of racism and homophobia; they are carried into everyday encounters unconsciously, no matter how vigilantly they are guarded 
against (for an extensive discussion of this issue, see Stevens, 2001). Inevitably, they connect to issues of personal identity. Internalised homophobia, for example, might reflect a transformed attack on the self originating from the earliest relationships in infancy, as much as society's prejudice. Experience of racial hatred might become available as a representation of earlier familial abuse.

Both dimensions, the social and the personal, must be addressed directly in class discussion and throughout the course, to prevent those trainees experiencing themselves as 'Other' from becoming more and more silent (Christian, Mokutu \& Rankoe, 2002). Whether or not adequate attention is paid to, and appropriate sensitivity is displayed towards the subjective (and very real) experience of feeling 'different' will affect the experience of the training. Some trainees have commented that attempts to be 'politically correct' have only ever felt false, untrustworthy, lacking in authenticity and served only to worsen the feelings of alienation whilst in training.

It has become evident through observation and discussion that a fragile, conflicted identity or self, one developmentally interrupted or disturbed, for whatever reasons, will be particularly predisposed towards feelings of fragmentation and identity loss. Such a person will struggle to hold on to, or find the emotional resilience that exists in those who have achieved a more securely integrated self. This will be particularly evident when confronted with a need to 'perform' whilst being observed - something that is implicit throughout the year and which is an unsettling experience for even the most securely integrated self.

Thus, there are certain constellations of self, which will be more vulnerable than others in this particular process, and for whom the clinical training, like the training of an anthropologist, is likely to be truly considered a shattering rite of death and rebirth (Wengle, 1987). However, whilst a clinical trainee's past will influence the experience, there are factors, in addition to unresolved developmental issues, which need to be taken into account in attempting to understand the kind of experience that each student has. These are fundamentally situated in a process designed to transform identity, rather than simply to build skills.

Given the ideas we have raised thus far, we have to ask how clinical trainees not only survive the process but also manage to transform themselves into the professionals that they ultimately become. How do they manage to function as if they have completed the third phase of the passage, whilst still very much in the second phase? What psychological strategies are used?

\section{Clinging to old values}

In his case study of Mandy Cesara, an anthropologist in the field, whose journey is in many ways parallel to that of clinical trainees, Wengle (1987) describes Cesara's desperate, and sometimes unrealistic, attempts to find some way to alleviate the feelings of alienation she was experiencing by maintaining a link with the past by clinging to something familiar, however small and apparently insignificant. One such 
example is found in her account of driving 150 miles to buy a cabbage. One clinical trainee described the way in which she repeatedly visited her former place of work during the early part of the course, deriving comfort from the familiar surroundings and conversations with colleagues. As she engaged emotionally with the training she found herself increasingly reluctant to visit and then she stopped going altogether.

If trainees are given offices and are encouraged to furnish them as they wish, some use this as a way of surrounding themselves with objects from their familiar, previous life. Others seem reluctant to make the office space their own until a number of weeks have passed, preferring to maintain a link with the old life by maintaining their study at home as the space in which they work. Significant and ongoing relationships also function as a familiar link with the identity that existed prior to entry into the marginal phase.

\section{In search of the local magistrate}

Wengle (1987) describes Mandy Cesara's relationship with a local magistrate upon whom she came to depend as she attempted to integrate herself into the local community. In our experience, one of the ways in which trainees attempt to manage the turmoil of the early part of their training is to form an idealising relationship (Wolf, 1988) with the person assigned to supervise their clinical work - their equivalent of Cesara's local magistrate. Trainees are expected to bring to their supervisor not only practical difficulties and theoretical questions, but also their struggles with their own feelings as they engage with the clinical work. Given the transitional space within which they find themselves and the destabilising of former identities, it is inevitable that they form a dependent relationship with supervisors, sometimes before they have even met. One trainee recalled not meeting his supervisor during the week in which the others in class had: 'I felt as if some part of my identity that everyone else had was missing. It was terrible!'

The supervisor-trainee relationship changes in the course of the year, as trainees' increasing sense of autonomy allows a gradual lessening of dependency and idealisation. Supervisors are called on less to check every interaction with clients and are engaged in broader issues of theory and ethics, and debate about appropriate intervention.

\section{The other local magistrate - the therapist}

The clinical trainee describing herself going 'right into pieces' went on to say: 'Somehow in every area there is too much. Thank goodness I am about to go to therapy perhaps some of it can be contained there.' Therapy potentially provides an important idealised and anchoring role for the initiate clinical psychologist. Therapy should be a safe and separate space in which to record and translate chaotic past and present experiences into something meaningful and transformational. Whether or not therapy 
can function in this way for trainees, depends on a number of particularly complex considerations.

Psychotherapy training programmes constructed along the principles advocated by Steiner (1984) build on three major sources of learning: (a) theory, (b) the actual experience of working with clients, together with personal supervision on each particular case and (c) personal therapy which would include discussion about what occurs in the spaces between the trainees and their clients, their fellow students, their instructors and, finally, between trainees and theory itself. There is often an expectation that trainees be in therapy, at least whilst they are in training, but preferably prior to the commencement of the course so that a relationship has already been established with the therapist. To form a new relationship whilst in this state of flux is obviously going to add to the struggle.

When Steiner advocates that a large component of the trainees' learning process should come out of their experience in therapy and their supervision, he is assuming that each is consistent with the other, as well as with the theoretical component of the course. Presumably, instructors accompanying initiates in rites of passage are able to provide a containing space because of a shared body of assumptions about the nature of the ritual and the world itself. Although the initiates are expected to fend for themselves, the authorities are clearly the authorities and know exactly what they want and need to convey. However, clinical trainees frequently encounter many contradictions between their personal psychotherapy experience and the models to which they are exposed in training. To extend the metaphor therefore, the therapist as a form of 'local magistrate' may be following a set of laws quite different from those acknowledged in the community.

Clearly the confusion surrounding the various models of intervention is likely to mean that instead of trainees' therapy performing a containing function, it could contribute to extensive personal and theoretical confusion, and serve therefore to undermine, rather than bolster, sorely needed self-esteem and confidence at this stage.

Appropriately, trainees' therapy is considered to be a sacred and private space. Unlike training analyses there is no communication set up between therapists and training staff. The choice of therapist is mostly left up to the trainee. Sometimes guidance is given to those who ask, with a list of names made available to them. It is quite clear that many have no idea what qualities they are looking for, nor what the purpose of the therapy is. Thus, choices are often made according to fees charged and time available, and not according to their own personal needs.

There are further potential problems involved in the requirement that the trainee be in therapy and the many and varied models taught, all occurring within the particular struggles involved in the marginal phase. We are referring here to the identity crisis involved during the training, which needs to be understood by the therapists involved. For example, if a first-year trainee is seeing someone who practices from a one-person, classical psychoanalytic model underpinned by the idea that patients are 
required to discover and ultimately renounce all their infantile desires (Mitchell, 1993), this could be painful and counterproductive for the individual in the marginal phase, in a training-induced state of infantile dependency.

\section{Sacra}

It is natural for clinical students to turn to written material and step-by-step guidelines constructed by trainers (sacra in the initiation literature) to illuminate their initiation journey. Wise men and women have gone before them, and their rich experience is captured in a multitude of articles, manuals, handouts and books, which they are urged to read both in preparation for clinical work and as a means of retrospective analysis of each clinical encounter.

The provision of sacra seems in some ways to be helpful as a way of containing trainees' anxiety about their first encounters with clients. However, it has two unintended negative effects. Firstly, it suggests to trainees that there are right and wrong ways of doing things, and that there is a 'how-to-do' manual to cover every eventuality. This truncates opportunities for trainees to draw on their own experience, problem-solving skills and creativity, while simultaneously encouraging an attitude of potentially mindless dependence on the thoughts of others, many of whom were not working in a South African context. Secondly, the inevitable moment arrives when clients say and do the unexpected. This unanticipated material, so different from what was rehearsed, easily confounds the trainees and leaves them either anxious about their capacity to do the work, or angry with the trainers for having anticipated wrongly what was in store for them. It is at this stage that trainees realise that, like the initiate and the anthropologist in training, they must find their way alone, and that reading and instruction will be of some, but limited assistance in the painful journey ahead. This is comparable to the expectation that once the rituals of separation have been performed, initiates find ways to bear the pain of transformation alone. It also parallels the experience of an anthropologist in the field, who suddenly finds himself or herself actually living in an exotic culture, engaging with a set of quite different cultural norms and having actually to speak the foreign language he or she so far only learnt theoretically.

Because of the defensive/protective and, therefore, containing function of theory, the expectation of being taught, as opposed to self-motivated learning, increases whenever trainees feel insecure and needy - around examinations, and seminar and case conference presentations, for example. Thus, paradoxically, the point at which there is nothing more to teach, is the point at which the absence of teaching, guidance and the giving of unambiguous sacra is most felt. Further, when the demand is not met, staff and supervisors will be experienced as withholding or uncaring: a regular and recurrent complaint. 
It is at these points in the training that, feeling abandoned by what are subjectively felt to be withholding instructors, trainees, with shock and disbelief, enter a state of flux and instability, a state in which they are expected to remain until they pass into the third phase, that is until they feel they can 'embark alone' (Quinodoz, 1996, p. 494). Paradoxically, trainees, whilst still feeling the insecurity characteristic of the second phase in their rite of passage, are expected to behave as if they have already passed through the next phase, that of 'reincorporation' when 'the ritual subject ... is in a relatively stable state once more' (Turner, 1969, p. 95).

\section{Communitas}

Despite their unstable state, trainees do, in fact, manage to do what is required of them. Consistent with their symbolic death, they feel that they are without status. According to the initiation literature, initiates in the marginal phase manage this through a sense of what Turner (1969) described as 'communitas', through which apparently solid bonds are formed. These are maintained throughout life, well after the rite of passage has been completed. There is evidence to suggest that clinical trainees identify strongly with one another, particularly in the first weeks of the course. Whilst the primary community is being formed, social activity is often important. One trainee remembered feeling that the year was going to be 'one long party. Quite a jolt to realise that it's not that way at all and we are going to have to get used to fitting in a bit of work between lunches!'

The expression of communitas sometimes involves a form of 'rebellion' in the class, which consolidates group cohesiveness. This is a common feature in this kind of training process (see for example, Rice, 1985). In our experience, it has taken a number of forms, the severity of which depends, at least in part, on the level of communitas involved. The rebellions have included refusal to carry out set tasks, complaints about the timetable, insisting on extending deadlines and occasionally major criticism of fundamental course features, such as examination format or staff. Whilst attempts at a group identity and the united complaints about the course and/or the staff continue throughout the year, they hardly ever sufficiently empower or contain the depth of the anxiety experienced by trainees in their struggle for renewal.

Thus seldom, if ever, does this attempt to merge and belong succeed in maintaining a cohesive identity throughout the marginal phase. It is common for tensions between members of the group to begin to emerge once the initial period of separation is over. Depending on the individuals involved in the course and their comfort with 'difference', some might complain about the lack of sensitivity to race, class or gender issues and dominant assumptions about, for example, the 'white' and/or 'heterosexual norm'. In relation to this, while discussion of race difference has historically been given authority by the struggle against racism in this country, gender difference and sexual orientation are less frequently legitimised as sites of struggle, even in more progressive circles (Levett \& Kottler, 1998). 
Interestingly, but not surprisingly, given the identity struggle involved, such differences become accentuated rather than overlooked, by the trainees themselves. Other values and cultural symbols therefore need to be found to contain the anxiety involved.

\section{Genera}

In a remarkably similar description to Bollas's idea of 'genera' (1993), Turner (1969) suggests that the initiates' freedom from structural restraints in the marginal phase, can give rise to novel thought, reflection and critical analyses. This is because ideas that had once been accepted uncritically are now thrown open to inspection. Having the psychic space to play with the theoretical and practical dimensions of the discipline is important, not only for the growth of the profession but for each trainee. To challenge theory, to question some or all of its assumptions, and to reach a personally satisfying modification of what has been handed down by predecessors is a powerful way of achieving a sense of belonging to a larger thinking community. Trainees are expected to arrive at a way of working that draws on a unique amalgam of their interpretation of theory and their own therapeutic style. However, a number of obstacles are placed in the way of their achieving this.

Most importantly, a course that places emphasis on learning through exposure to plentiful clinical work tends to leave little time for theoretical debate. Trainees are certainly exposed to theory, but often this is the context for learning specific skills or therapeutic techniques immediately required for use in specific clinical work. Trainees quickly become unsettled when their teachers introduce critical perspectives on theoretical models. For example, they resist debate about heterosexist or Northern Hemisphere assumptions in many models of family therapy, at moments when they are looking for unambiguous direction in a family therapy intervention. To contain their anxiety, they need both the theory and the tools it generates to be credible - and learnable. Moreover, clinical courses tend to be packed with seminars, case conferences, written and practical assignments, field trips, community projects, as well as ongoing casework and supervision. All this leaves little time for theoretical reflection. In fact, a recurrent complaint in UCT's course is that there is never enough time to read widely.

Another problem lies in the nature of the theory itself. The shadow of Freud's legacy stretches into many aspects of our work, and the common practice of studying seminal articles of Freud, Anna Freud, Klein and other psychoanalytic luminaries, instils in trainees a sometimes inappropriate reverence for these 'sacred' texts. It is a brave trainee who challenges and seeks to modify this body of theory, precisely because it feels like a sacrilegious act. The atmosphere of reverence and mystique surrounding psychoanalytic theory creates a dynamic in which other more flexible and fluid bodies of theory - those in which debate is welcomed - may become undervalued and therefore less prized as tools of the trade. 
Another difficulty that often impedes the creation of a space for thoughtful play in the training concerns the dual and contradictory role of staff being called on to both supervise and examine. This flies in the face of the majority of psychotherapy training courses, which require that neither therapists nor supervisors be in any way connected to the examining body. It is generally accepted that supervision should take place in a relaxed atmosphere (Buirski \& Haglund, 2001; Fuqua, 1994) so as to encourage frank and free discussion about counter-transferential issues, self-disclosure and anything else that trainees might feel is problematic in the work they are required to do. This is, after all, one of the major sources of training - through the experience of 'ample clinical experience' (Steiner, 1984, p. 56). However, if the supervisor is likely to be an examiner, clearly there will be pressure to demonstrate competence in received models, as opposed to taking the risk of experimenting with new perspectives.

Case conferences and seminars should serve a similar safe teaching space, but again, those present are likely to examine later in the year. This too then becomes a potentially persecutory or narcissistically injuring space rather than one that fosters growth and creativity. In addition to the presence of the staff at case conferences, trainees are performing in front of their peers. If there are difficult dynamics between them that may or may not relate to competition (which is fostered in the university system, particularly where large numbers of students compete for few places in postgraduate courses) this will constrict the learning process. The inevitable comparisons make trainees particularly cautious about sharing their creativity, for fear of being narcissistically injured or shamed. They are likely to protect themselves by negotiating around obstacles rather than addressing them (Mollon, 1989), particularly if the space does not feel safe enough, as is surely the case, given what we have said above.

Thus one of the most important aspects of supervision and teaching can be lost, namely the creation of a 'space for thinking - a kind of thinking which is more akin to maternal reverie, as described by Bion, than problem solving' (Mollon, 1989, p. 113).

\section{CONCLUDING OBSERVATIONS AND RECOMMENDATIONS}

In this article, we have described a marginal psychological state that accompanies early periods of training for clinical psychologists. We have argued that this may be a necessary phase in the acquisition of a new identity, as old ways of seeing, knowing and performing are, over a short period of time, replaced with the new and unfamiliar. We have also suggested that in this anxiety-provoking marginal frame, trainers might unwittingly contribute to the already unstable and vulnerable state of trainees, largely through the ambiguity and contradictions inherent in the demands made on them. Our concern is that there is a fine line between a creative marginality in which psychological structure is fluid enough to accommodate substantial change and a potentially more damaging state in which little creative learning is possible. 
Our examination of the training process leads us to a number of conclusions, all of which may translate into recommendations for future training programmes.

Clinical psychology training programmes demand of trainees a fundamental level of personal engagement with the process, including self-questioning, psychotherapy, and self-disclosure within peer and supervisory settings. The training therefore entails more than simply the acquisition of knowledge, skills, and experience; it asks for a shift in identity. Like initiation, the process of acquiring the new identity takes place in the phases of separation, living in the margins and reintegration.

The marginal state is accompanied by feelings of unfamiliarity, anxiety, helplessness and disorientation. The work is separated from general discussion in the outside world of friends and family by its confidential nature. It is, however, intensely preoccupying, partly because of the responsibility and strength of feeling it carries with it, and partly because of its unfamiliarity. In the marginal state of having left the old, and before becoming 'adult' psychologists, trainees need guides, and these are often found in the form of idealised relationships with supervisors and therapists. The class also forms a community within which the struggle with the new identity is shared and supported. Theoretical knowledge and practical rules are helpful insofar as they provide containing structures with which clinical trainees, like initiates can begin to find their feet.

Difficulties in the rite of passage concern the essential ambiguity of the marginal phase, a time during which trainees are expected to shoulder clinical responsibilities even though they experience themselves as insufficiently knowledgeable. This ambiguity is compounded by mixed messages from teachers, who often find it difficult to reach a balance between giving minute, blow-by-blow instructions and giving no instructions at all. Trainees are, at times, clearly marked as students who are being closely watched and supervised. At other times, they are treated as relatively responsible colleagues. Further confusion is caused by offering a mixture of theories as sacra to guide the experience. Trainees also struggle to become confident theoretically, partly because of the lack of time they have to read and partly because there are few free spaces in which to have theoretical play.

If these are indeed the challenges facing trainees, what then constitutes an adequate training environment? Clearly the 'good-enough' training will cover a variety of diverse models. However, as with judgements about good-enough mothering, there are minimum standards to be set. The comments that follow arise from our structural analysis and will hopefully begin a dialogue with colleagues broadly in the area of ethical practice in psychology training.

Firstly, clinical psychology training is likely to remain a form of identity transformation, simply because of the nature of the work, which is often in the realm of the painful and the private. Unless it is recognised, labelled and adapted to, such transformation is anxiety-provoking and potentially damaging to the trainee. Trainers, we believe, will be most helpful when they expect a degree of dependence, disorientation and confusion, and prepare themselves to contain this in ways that will not be 
experienced as shaming. This is particularly pertinent to feedback processes during the course and after examinations, when every care should be taken to separate commentary on personality style from work performance and evaluation. It is often important to point out to trainees their counter-transferential blindnesses, but these should then be referred to their personal therapy for further discussion.

Identity flux brings with it uncertainty and changes in self-esteem. It is important that trainees be encouraged to recognise, and build on, the knowledge, experience and skills they brought with them into the training as a means of self-esteem regulation. The challenge in this for trainers is to embrace the idea that to empower trainees in this way does not abrogate either their own power or their authority.

Supervisors are central to the steady acquisition of a new identity. Their role as guides and mentors weaves a delicate line between the negotiation of personal, social and professional identities. This ambiguity will always be there, but its necessary presence makes it imperative that the difference between therapy and supervision should be held in mind at all times. Moreover, for supervisors to act as evaluators might easily be experienced as a betrayal of trust, given the degree of vulnerability and openness required in the relationship. Therefore, maintaining some separation between supervision and evaluation in the training process seems essential.

In a number of South African courses, trainees are exposed to cases that would challenge even very experienced clinicians. These are public clinic cases involving multiple trauma, including poverty, poor school resources, violence, loss and physical illness. To work alongside the trainee as co-therapist, sharing the burden of the responsibility is one way of providing structure and support. Having a supervisor always on hand while casework is under way is another. The trainee needs to feel empowered to do the work, but not to feel alone in it. The challenge to trainers is therefore to involve themselves directly in the clinical work they ask trainees to undertake, not simply to comment on it from a distance.

Finally, the marginal state, potentially, is a creative one and a crucible within which new theory, appropriate to South African settings, can be developed. Training therefore might fruitfully be seen as a transformational opportunity, not only for the trainees, but also for the profession as a whole. This would require those involved with training to balance transmission of well-established theory with experimentation along less well-trodden paths.

\section{REFERENCES}

Barnett, R. and Hyde, J. (2001). Women, men, work, and family: An expansionist theory. American Psychologist, 56, 781-796.

Bollas, C. (1993). Being a character. London: Routledge

Buirski, P. and Haglund, P. (2001). Making sense together: The intersubjective approach to psychotherapy. Northvale, NJ: Jason Aronson.

Christian, R., Mokutu, M. and Rankoe, M. (2002). Black students' experiences of training at a 'white' institution. In L. Swartz, K. Gibson and T. Gelman (Eds), Reflective practice: 
Psychodynamic ideas in the community (pp.33-44). Cape Town: Human Research Council Publications.

Clyde Mitchell, J. (2000). Case and situation analysis. In R. Gomm, M. Hammersley and P. Foster (Eds), Case study method (pp. 165-186). London: Sage.

Duncan, N., van Niekerk, A., de la Rey, C. and M. Seedat (Eds) (2001). 'Race', racism, knowledge production and psychology in South Africa. New York: Nova Science Publishers.

Fuqua, P.B. (1994). Teaching, learning and supervision. In A. Goldberg (Ed.), A decade of progress: Progress in self psychology, Vol. 10 (pp. 79-97). Hillsdale, NJ: Analytic Press.

Levett, A. and Kottler, A.E. (1998). Through a lens, darkly. In E. Burman (Ed.), Deconstructing feminist psychology (pp. 184-205). London: Sage.

Mitchell, S.A. (1993). Hope and dread in psychoanalysis. New York: Basic Books.

Mollon, P. (1989). Anxiety, supervision and a space for thinking: Some narcissistic perils for clinical psychologists in learning psychotherapy. British Journal of Medical Psychology, $62,113-122$.

Quinodoz, J.-M. (1996). The sense of solitude in the psychoanalytic encounter. International Journal of Psycho-Analysis, 77, 481-496.

Rice, C.A. (1985). The fights of Spring: Separation, individuation, and grief in training centres. Psychotherapy, 22(1), 97-100.

Seedat, M., Duncan, N. and Lazarus, S. (Eds) (2001). Community psychology: Theory, method and practice. Cape Town: Oxford University Press.

Steiner, J. (1984). The training of psychotherapists. Psychoanalytic Psychotherapy, 1, 55-63.

Stevens, G. (2001). Racism and cultural imperialism in the training of black psychologists in South Africa: Identity, ambiguity and dilemmas of praxis. In N. Duncan, A. van Niekerk, C. de la Rey and M. Seedat (Eds), 'Race', racism, knowledge production and psychology in South Africa (pp. 45-60). New York: Nova Science Publishers.

Stryker, S. and Burke, P. (2000). The past, present and future of identity theory. Social Psychology Quarterly, 63, 284-297.

Suffla, S., Stevens, G. and Seedat, M. (2001). Mirror reflections: The evolution of organised professional psychology in South Africa. In N. Duncan, A. van Niekerk, C. de la Rey and M. Seedat (Eds), 'Race', racism, knowledge production and psychology in South Africa (pp. 27-36). New York: Nova Science Publishers.

Swartz, L. (1998). Culture and mental health: A Southern African view. Cape Town: Oxford University Press.

Swartz, L., Gibson, K. and Gelman, T. (Eds) (2002). Reflective practice: Psychodynamic ideas in the community. Cape Town: Human Research Council Publications.

Tajfel, H. (1979). Individuals and groups in social psychology. British Journal of Social and Clinical Psychology, 18, 183-191.

Turner, V. (1967). The forest of symbols. Ithaca and London: Cornell University Press.

- (1969). The ritual process. London: Routledge and Kegan Paul.

Wengle, J. (1987). Death and rebirth in fieldwork: A case study. Culture, Medicine and Psychiatry, 11, 357-385.

Wolf, E.S. (1988). Treating the self: Elements of clinical self psychology. New York: Guilford Press. 